\title{
Genetics of "high-risk" chronic lymphocytic leukemia in the times of chemoimmunotherapy
}

\author{
Alexander Ring and Thorsten Zenz \\ Department of Medical Oncology and Haematology, University Hospital Zurich and University of Zurich, Zurich, Switzerland \\ E-mail: THORSTEN ZENZ - thorsten.zenz@usz.ch
}

doi:10.3324/haematol.2020.246504

C hronic lymphocytic leukemia (CLL) is a common leukemic B-cell lymphoma driven by distinct molecular features such as autonomous B-cell receptor signaling and genetic alterations including mutations targeting the DNA damage machinery, RNA processing and splicing, oncogenic signaling pathways (such as Notch) as well as epigenetic and chromatin modification. ${ }^{1-3}$ In a simplified model, the "sum" of autonomous Bcell receptor signaling and driver mutations govern CLL progression. In addition, mutations in individual genes, such as $T P 53^{4,5}$ are tightly linked to refractoriness to chemotherapy. ${ }^{6}$ This model summarizes current knowledge, but we cannot exclude the possibility that additional (maybe unknown) mechanisms contribute significantly to proliferative drive and may thus predispose (or select for?) driver mutations. The current emergence of additional data and more appropriate statistical tools to query complex molecular data can be expected to provide novel insights into the pathogenesis of CLL. ${ }^{\text {? }}$

The study by Jennifer Edelmann and colleagues published in this issue of Haematologica is an informative addition to the catalogs of gene mutations in CLL. In this study, Edelmann and colleagues use cohorts of patients treated with chemotherapy/alemtuzumab in multiple trials and high-resolution single nucleotide polymorphismarray profiling and sequencing to characterize copy number variants and a limited mutational landscape of highrisk CLL cases. ${ }^{8}$ The analysis summarizes data from 146 patients from CLL trials (CLL8, CLL11, CLL20), in which high risk was defined as either a TP53 deletion/mutation genotype, "complex" karyotype/ increased genomic complexity or purine-analog refractory cases (progression-free survival $<6$ months). The authors thus provide a comprehensive description of genomic alterations in high-risk CLL patients that are selected for in the context of chemo(immuno)therapy, by building groups and individually testing for unbalanced incidences of mutations. The results lead to a description of well-known tumor drivers, which appear to contribute to high-risk CLL in addition to TP53; MYC, [gain (8)(q24)], CDKN2A/B [del(9)(p21)] and Notch pathway mutations.

The authors describe mutations in Notch-associated genes and known negative regulators (i.e. SPEN, RBPJ). They found that the above genes were mutated/deleted in $3.7-8.2 \%$ of high-risk CLL patients and showed that mutated cases had higher levels of expression of Notch target genes (e.g. HES1, DTX1 and MYC). Furthermore, they revealed that $S N W 1$ is a potential negative regulator of the Notch signaling pathway. SNW1 has also been shown to act as a co-activator of Notch-driven transcription. $^{9}$

Notch signaling is an evolutionarily conserved signaling pathway that allows cell-cell interactions regulating a wide range of biological functions. ${ }^{10}$ There are four mammalian members of NOTCH transmembrane proteins or receptors (NOTCH1 - 4) which have only partially overlapping functions despite similar structures. These receptors function as ligand-activated transcription factors, interacting with transmembrane ligands (Delta-like1, 3 and 4, and Jagged1 and 2) (Figure 1A, B).

While Notch signaling plays an important physiological role in hematopoiesis and hematopoietic stem cell biology, ${ }^{11,12}$ aberrant Notch signaling has been found to be an oncogenic driver in precursor lymphoid and myeloid neoplasms as well as mature B-cell neoplasms with different mechanisms of oncogenic pathway activation including mutations in Notch receptors, mutations in negative regulators (e.g. $F B X W 7$ ) or overexpression of ligands and receptors. ${ }^{13-15}$ NOTCH1 is one of the most frequently mutated genes in CLL, ${ }^{16}$ affecting approximately $12 \%$ of cases. ${ }^{17,18}$ The majority of mutations occur in coding regions leading to stabilization of the Notch intracellular domain (NICD) via loss of the PEST [proline (P), glutamic $\operatorname{acid}(\mathrm{E})$, serine $(\mathrm{S})$, and threonine $(\mathrm{T})$ ] domain. NOTCH1 gain-of-function mutations in CLL were first described by Ianni et al. ${ }^{19,20}$ and were later found in large-scale sequencing studies. ${ }^{21,22}$ Additionally, mutations in the non-coding 3' untranslated region have been described. ${ }^{17,23}$ These mutational events ultimately lead to the accumulation of NCID and increase Notch signaling activity. Notch activation through mechanisms other than activating mutations also frequently occur in CLL. ${ }^{18}$ with roughly $50 \%$ of CLL cases exhibiting high levels of NICD without detectable NOTCH1 mutations. ${ }^{18,20}$ Although potential mechanisms of NOTCH1 mutation-independent pathway activation have been proposed (e.g. MED12 mutations $^{24}$ ), the biology remains incompletely understood. Mutations in the negative regulator FBXW7 have been described in CLL. ${ }^{25}$

NOTCH1 has been found to be an adverse prognostic marker in $\mathrm{CLL}^{26-29}$ and has been associated with the cooccurrence of other adverse prognostic factors in CLL, such as IGHV mutational status ${ }^{30}$ and trisomy $12 .{ }^{31}$ While NOTCH1 mutations are more frequently found in CLL with unmutated IGHV, the accumulation of NICD without NOTCH1 mutations seems similarly distributed in CLL with unmutated and mutated IGHV genes. ${ }^{18}$ Integration of information about the presence or absence of NOTCH1 mutations into prognostic scoring systems improved survival predictions. ${ }^{32}$ NOTCH1 mutations have not only been linked to progressive disease, but also to the earliest stages of development of CLL. ${ }^{33}$

Current approaches targeting Notch signaling include $\gamma$ secretase inhibitors, which block the proteolytic cleavage of NICD. More than $100 \gamma$-secretase inhibitors have been developed, ${ }^{34}$ with some demonstrating effects in CLL as single agents or in combination with other drugs. ${ }^{35,36}$ Monoclonal antibodies targeting Notch receptors (e.g. OMP-52M51) have been tested in pre-clinical ${ }^{137}$ and clinical 


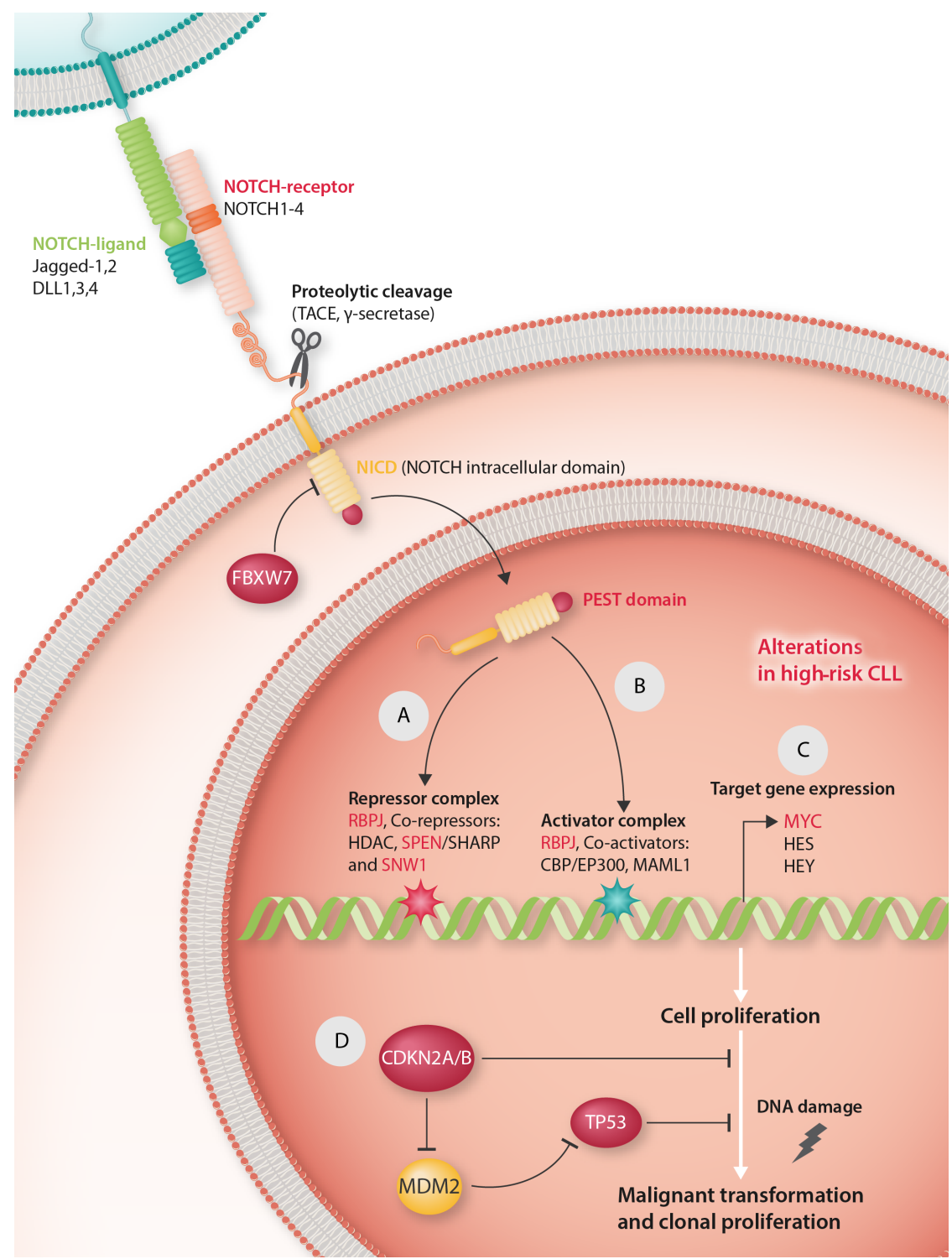

Figure 1. Molecular drivers of high-risk chronic lymphocytic leukemia. (A, B) Notch signaling. In its inactive state the Notch transcriptional complex is bound by co-repressors such as SPEN, histone deacetylases (HDAC) and, potentially, SNW1 (A). Binding of Notch ligands (Jagged-1,2, DLL1, 3, 4) to Notch receptors leads to proteolytic cleavage of the intracellular domain (NICD) via $\gamma$-secretase and translocation of NICD to the nucleus to form a transcriptionally active complex with MAML1 (Mastermind-like protein 1), RBPJ (Recombination signal binding protein for immunoglobulin kappa $J$ region) and transcriptional co-activators such as the histone acetyl transferases CBP/EP300, leading to Notch target gene expression (including MYC, HES, HEY) (B). Termination of Notch signaling is achieved mainly via ubiquitination of the PEST degradation domain of NICD by FBXW7 (F-box/WD repeat-containing protein 7). (C) Alterations in MYC activity. MYC is a direct target of Notch signaling driving cell proliferation. Gain of the MYC locus (8)(q24) enhances activity. (D) DNA damage checkpoint. TP53 is frequently altered and a hallmark of high-risk chronic lymphocytic leukemia (CLL). Loss of function in CDKN2A/B impairs TP53 tumor suppressor function and cell cycle control. (Gene symbols and gene names in red represent altered/mutated genes in high-risk CLL).

studies (NCT01778439, NCT 01703572). Indirect targeting approaches are also under investigation (e.g. bepridil). ${ }^{38}$

Dysregulation of MYC in B-cell tumors has been well established and comprehensively reviewed ${ }^{39,40}$ Edelmann et al. have demonstrated that gain of the MYC locus [gain (8)(q24)] frequently occurs in high-risk CLL. MYC has also been shown to be a direct target of $\mathrm{NOTCH}^{41}$ (Figure 1C).

Disruption of the DNA damage repair complex and associated cell cycle control or arrest is a hallmark of highrisk CLL. The protein products of both CDKN2A and CDKN2B (i.e. p16INK4a, p14ARF and p15INK4b, respectively) are central to DNA damage-related cell cycle control by interacting with both p53 and RB1 as well as direct inhibitors of cyclin-dependent kinases (CDK). Both p16INK4a and p15INK4b inhibit CDK4 and CDK6 and lead to the activation of RB1. ${ }^{42}$ The alternate reading frame product p14ARF inhibits MDM2, thereby stabilizing p53. ${ }^{43}$ (Figure 1D). These mechanisms have important tumor suppressor function guarding against DNA damage with potentially tumorigenic mutations and loss of these tumor suppressors may exert deleterious effects similar to loss of TP53. Cases of transformed CLL into aggressive lymphoma (Richter transformation) often exhibit CDKN2A/B disruption. ${ }^{44}$

With the advent of chemotherapy-free treatments, it remains to be seen if the results presented will be applicable to current standards of care. For a more comprehensive understanding of CLL, clonal evolution and predictive markers, future studies will leverage comprehensive protein, methylation and RNA expression in addition to DNA-level investigations in a genome-wide manner. As these data emerge and are analyzed with more complex statistical models ${ }^{7}$ the mechanisms underlying aggressive disease will become clearer. We hope this will have direct implications for the clinical management of CLL patients. One simple step in this direction is an open approach to data sharing and access, a prerequisite to advance knowledge on rare variants. We are certain that the study groups involved in the analysis by Edelmann et al. will also take a lead in this area. 


\section{References}

1. Baliakas P, Jeromin S, Iskas $M$, et al. Cytogenetic complexity in chronic lymphocytic leukemia: definitions, associations, and clinical impact. Blood. 2019;133(11):1205-1216.

2. Burger JA, Chiorazzi N. B cell receptor signaling in chronic lymphocytic leukemia. Trends Immunol. 2013;34(12):592-601.

3. Landau DA, Tausch E, Taylor-Weiner AN, et al. Mutations driving CLL and their evolution in progression and relapse. Nature. 2015;526(7574):525-530.

4. Zenz T, Eichhorst B, Busch R, et al. TP53 mutation and survival in chronic lymphocytic leukemia. J Clin Oncol. 2010;28(29):4473-4479.

5. Zenz T, Kröber A, Scherer K, et al. Monoallelic TP53 inactivation is associated with poor prognosis in chronic lymphocytic leukemia: results from a detailed genetic characterization with long-term follow-up. Blood. 2008;112(8):3322-3329.

6. Dietrich S, Oleś M, Lu J, et al. Drug-perturbation-based stratification of blood cancer. J Clin Invest. 2018;128(1):427-445.

7. Argelaguet R, Velten B, Arnol D, et al. Multi-omics factor analysisa framework for unsupervised integration of multi-omics data sets. Mol Syst Biol. 2018;14(6):e8124

8. Edelmann J, Holzmann K, Tausch E, et al. Genomic alterations in highrisk chronic lymphocytic leukemia frequently affect cell cycle key regulators and NOTCH1-regulated transcription. Haematologica. 2020; Volume 105(5):1379-1390.

9. Hong $\mathrm{M}, \mathrm{He} \mathrm{J}$, Li S. SNW1 regulates Notch signaling in neuroblastoma through interacting with RBPJ. Biochem Biophys Res Commun. 2019;509(4):869-876.

10. Fortini ME. Notch sgnaling: the core pathway and its posttranslational regulation. Dev Cell. 2009;16(5):633-647.

11. Pajcini KV, Speck NA, Pear WS. Notch signaling in mammalian hematopoietic stem cells. Leukemia. 2011;25(10):1525-1532.

12. Kojika S, Griffin JD. Notch receptors and hematopoiesis. Exp Hematol. 2001;29(9):1041-1052

13. Kridel R, Meissner B, Rogic S, et al. Whole transcriptome sequencing reveals recurrent NOTCH1 mutations in mantle cell lymphoma. Blood. 2012;119(9):1963-1971.

14. Lee SY, Kumano K, Nakazaki K, et al. Gain-of-function mutations and copy number increases of Notch2 in diffuse large B-cell lymphoma. Cancer Sci. 2009;100(5):920-926.

15. Karube K, Martínez D, Royo C, et al. Recurrent mutations of NOTCH genes in follicular lymphoma identify a distinctive subset of tumours. J Pathol. 2014;234(3):423-430.

16. Rosati E, Baldoni S, De Falco F, et al. NOTCH1 aberrations in chronic lymphocytic leukemia. Front Oncol. 2018;8:229.

17. Puente XS, Beà S, Valdés-Mas R, et al. Non-coding recurrent mutations in chronic lymphocytic leukaemia. Nature. 2015;526(7574): 519-524.

18. Fabbri $G$, Holmes $A B$, Viganotti $M$, et al. Common nonmutational NOTCH1 activation in chronic lymphocytic leukemia. Proc Natl Acad Sci U S A. 2017;114(14):E2011-E2919.

19. Ianni M Di, Baldoni S, Rosati E, et al. A new genetic lesion in B-CLL: A NOTCH1 PEST domain mutation. Br J Haematol. 2009;146(6): 689-691.

20. Rosati E, Sabatini R, Rampino G, et al. Constitutively activated Notch signaling is involved in survival and apoptosis resistance of BCLL cells. Blood. 2009;113(4):856-865

21. Fabbri G, Rasi S, Rossi D, et al. Analysis of the chronic lymphocytic leukemia coding genome: role of NOTCH1 mutational activation. J Exp Med. 2011;208(7):1389-1401

22. Puente XS, Pinyol M, Quesada V, et al. Whole-genome sequencing identifies recurrent mutations in chronic lymphocytic leukaemia. Nature. 2011;475(7354):101-105.

23. Bittolo T, Pozzo F, Bomben R, et al. Mutations in the $3^{\prime}$ untranslated region of NOTCH1 are associated with low CD20 expression levels in chronic lymphocytic leukemia. Haematologica. 2017;102(8):e305 e309.

24. Wu B, Słabicki M, Sellner L, et al. MED12 mutations and NOTCH signalling in chronic lymphocytic leukaemia. Br J Haematol. 2017;179(3):421-429.

25. Close V, Close W, Kugler SJ, et al. FBXW7 mutations reduce binding of NOTCH1, leading to cleaved NOTCH1 accumulation and target gene activation in CLL. Blood. 2019;133(8):830-839.

26. Rossi D, Rasi S, Fabbri G, et al. Mutations of NOTCH1 are an independent predictor of survival in chronic lymphocytic leukemia. Blood. 2012;119(2):521-529.

27. Stilgenbauer S, Schnaiter A, Paschka P, et al. Gene mutations and treatment outcome in chronic lymphocytic leukemia: results from the CLL8 trial. Blood. 2014;123(21):3247-3254.

28. Villamor N, Conde L, Martínez-Trillos A, et al. NOTCH1 mutations identify a genetic subgroup of chronic lymphocytic leukemia patients with high risk of transformation and poor outcome. Leukemia. 2013;27(5):1100-1106.

29. Larrayoz M, Rose-Zerilli MJJ, Kadalayil L, et al. Non-coding NOTCH1 mutations in chronic lymphocytic leukemia; their clinical impact in the UK CLL4 trial. Leukemia. 2017;31(2):510-514.

30. López C, Delgado J, Costa D, et al. Clonal evolution in chronic lymphocytic leukemia: Analysis of correlations with IGHV mutational status, NOTCH1 mutations and clinical significance. Genes Chromosom Cancer. 2013;52(10):920-927.

31. Balatti V, Bottoni A, Palamarchuk A, et al. NOTCH1 mutations in CLL associated with trisomy 12. Blood. 2012;119(2):329-331.

32. Döhner H, Stilgenbauer S, Benner A, et al. Genomic aberrations and survival in chronic lymphocytic leukemia. $N$ Engl J Med. 2000;343(26):1910-1916.

33. Damm F, Mylonas E, Cosson A, et al. Acquired initiating mutations in early hematopoietic cells of CLL patients. Cancer Discov. 2014;4(9):1088-1101

34. Rizzo P, Osipo C, Foreman K, Golde T, Osborne B, Miele L. Rational targeting of Notch signaling in cancer. Oncogene. 2008;27(38):51245131

35. Rosati E, Sabatini R, De Falco F, et al. $\gamma$-Secretase inhibitor I induces apoptosis in chronic lymphocytic leukemia cells by proteasome inhibition, endoplasmic reticulum stress increase and notch down-regulation. Int J Cancer. 2013;132(8):1940-1953.

36. López-Guerra M, Xargay-Torrent S, Rosich L, et al. The $\gamma$-secretase inhibitor PF-03084014 combined with fludarabine antagonizes migration, invasion and angiogenesis in NOTCH1-mutated CLL cells. Leukemia. 2015;29(1):96-106.

37. Wu Y, Cain-Hom C, Choy L, et al. Therapeutic antibody targeting of individual Notch receptors. Nature. 2010;464(7291):1052-1057.

38. Baldoni S, Del Papa B, Dorillo E, et al. Bepridil exhibits anti-leukemic activity associated with NOTCH1 pathway inhibition in chronic lymphocytic leukemia. Int J Cancer. 2018;143(4):958-970.

39. Nguyen L, Papenhausen P, Shao H. The role of c-MYC in B-cell lymphomas: diagnostic and molecular aspects. Genes (Basel). 2017;8(4).

40. Huh YO, Lin KIC, Vega F, et al. MYC translocation in chronic lymphocytic leukaemia is associated with increased prolymphocytes and a poor prognosis. Br J Haematol. 2008;142(1):36-44.

41. Weng AP, Millholland JM, Yashiro-Ohtani Y, et al. c-Myc is an important direct target of Notch1 in T-cell acute lymphoblastic leukemia/lymphoma. Genes Dev. 2006;20(15):2096-2109.

42. Ruas M, Peters G. The p16(INK4a)/CDKN2A tumor suppressor and its relatives. Biochim Biophys Acta. 1998;1378(2):F115-177.

43. Prives C, Hall PA. The P53 pathway. J Pathol. 1999;187(1):112-126.

44. Fabbri G, Khiabanian $H$, Holmes AB, et al. Genetic lesions associated with chronic lymphocytic leukemia transformation to Richter syndrome. J Exp Med. 2013;210(11):2273-2288. 